Revue d'histoire de l'Amérique française

REVUE D.HISTOIRE DE L'AMÉRIQUE FRANÇAISE

McROBERTS, Kenneth, dir., Beyond Quebec. Taking Stock of Canada (Montréal, McGill-Queen's University Press, 1995), $435 \mathrm{p}$.

\title{
Sarah Fortin
}

Volume 50, numéro 2, automne 1996

URI : https://id.erudit.org/iderudit/305536ar

DOI : https://doi.org/10.7202/305536ar

Aller au sommaire du numéro

Éditeur(s)

Institut d'histoire de l'Amérique française

ISSN

0035-2357 (imprimé)

1492-1383 (numérique)

Découvrir la revue

Citer ce compte rendu

Fortin, S. (1996). Compte rendu de [McROBERTS, Kenneth, dir., Beyond Quebec. Taking Stock of Canada (Montréal, McGill-Queen's University Press, 1995),

435 p.] Revue d'histoire de l'Amérique française, 50(2), 297-299.

https://doi.org/10.7202/305536ar d'utilisation que vous pouvez consulter en ligne.

https://apropos.erudit.org/fr/usagers/politique-dutilisation/ 
McROBERTS, Kenneth, dir., Beyond Quebec. Taking Stock of Canada (Montréal, McGill-Queen's University Press, 1995), $435 \mathrm{p}$.

Beyond Quebec est issu d'une conférence organisée par le Robarts Centre for Canadian Studies de l'Université York au printemps 1994, laquelle avait pour objet de faire le point sur le Canada hors-Québec et d'évaluer les conséquences que pourrait avoir la souveraineté du Québec pour le reste du Canada. L'ouvrage réunit les contributions de vingt-deux universitaires provenant des quatre coins du Canada hors-Québec, spécialistes des sciences humaines et sociales, des arts et des lettres.

D'entrée de jeu, il faut souligner l'intérêt et l'utilité d'un ouvrage qui examine la nature, la cohésion et les caractéristiques du Canada hors-Québec. Mises à part les réflexions de Philip Resnick $(1990,1991,1994)$ et la publication du collectif «English Canada» Speaks Out en 1991, très peu d'attention avait été consacrée à ces questions jusqu'à maintenant. Vue la qualité des analyses et des observations, il y a tout lieu de croire que Beyond Quebec deviendra rapidement un passage obligé pour qui veut s'initier à l'expérience canadienne hors-Québec, dans ses dimensions régionale et culturelle en particulier.

Trop souvent mise de côté, la culture cette fois n'est pas en reste puisque toute une section lui est consacrée. Bien que la musique, la danse et la peinture ne soient pas abordées, cette question occupe tout de même plus du quart de l'ouvrage, tant en termes de chapitres (6/22) que de pages. C'est là une décision significative et sans doute l'apport le plus original de cette conférence. On y trouve un bilan critique et un portrait de la scène culturelle canadienne hors-Québec (littérature, télévision, édition, cinéma, institutions culturelles, arts autochtones) qui révèlent les tensions qui traversent ce secteur d'activités et identifient les défis qu'il doit relever. Les problèmes qui caractérisent habituellement la scène politique y sont abordés sous l'angle de la culture: l'utilité des politiques nationales, la menace et la résistance à l'américanisation, la quête identitaire, l'espace laissé au multiculturalisme et aux autochtones, l'impérialisme de l'économie de marché, etc.

Quatre autres sections sont consacrées au sentiment national (qui inclut trois chapitres sur les régions), à l'économie (l'intégration continentale), à la société (les femmes, les minorités linguistiques et culturelles, les autochtones) et au futur du Canada hors-Québec.

Le portrait ainsi tracé est multiforme. On nous présente un Canada hétérogène sur tous les plans. Il y a bien sûr le Canada des régions (problématique centre-périphérie), mais aussi celui des clivages politiques et idéologiques qui opposent les «chartistes» aux individualistes, les nationalistes aux continentalistes, les partisans de l'intervention et ceux du laisser-faire, les minorités à la majorité, etc. L'impression est renforcée par les divergences d'opinion qui opposent plusieurs analystes, notamment en ce qui concerne l'autonomie du Canada par rapport à son voisin américain - les textes de Frank Davey et de Rowland Lorimer sur le milieu littéraire et le monde de l'édition ou ceux de Stephen Clarkson et de Melville L. McMillan sur l'ave- 
nir économique, par exemple. Compte tenu de la tendance que nous avons au Québec à présumer de l'homogénéité du Canada hors-Québec, il me semble important de souligner ce pluralisme.

Une pluralité qui n'empêche pas, cependant, la cohésion sociale et politique de se manifester. En effet, même si le reste du Canada est tiraillé par les intérêts divergents de ses composantes, la plupart des participants concluent à l'existence d'un sentiment d'appartenance collectif qui rappelle la société globale de Simon Langlois. À cet égard, on aurait aimé retrouver un chapitre portant plus spécifiquement sur l'émergence et le développement d'une identité et d'un imaginaire canadiens, qui aurait pu explorer le rôle des institutions sociales et politiques ou celui des sciences sociales.

En regard de ce constat généralisé qui révèle un Canada uni dans la diversité, il n'est peut-être pas tellement étonnant de voir que la sécession du Québec n'est pas considérée, à trois exceptions près, comme pouvant altérer substantiellement la réalité politique, économique et culturelle du reste du Canada. Seuls Stephen Clarkson pour qui «The rest of Canada could well have enough cultural substance and political coherence, but it would have neither the economic viability nor the international raison d'être to form a successful nation» (p. 270), Phyllis Leblanc, qui rappelle les défis que poserait le départ du Québec pour les francophones ailleurs au Canada et Thomas Courchesne, selon qui le départ du Québec renforcerait l'américanité du reste du Canada, se démarquent de ce consensus. Les changements probables dans la configuration des clivages politiques (F. L. Morton), les coûts de transition et la séparation physique des provinces maritimes (Robert Finbow), les scénarios possibles en matière de réforme institutionnelle (Roger Gibbins) sont bien évoqués, mais en définitive le départ du Québec — ou sa présence n'est pas une variable décisive dans l'évolution du reste du Canada.

Les deux grands arguments évoqués pour justifier ces opinions - sur le plan interne, la cohésion et l'autonomie qui caractérisent actuellement les deux sociétés, particulièrement dans le monde culturel; sur le plan externe, les forces politiques et économiques globales qui influent déjà sur la dynamique politique et économique - ne sont pas sans rappeler les raisonnements mis de l'avant pour appuyer la souveraineté du Québec.

D'un point de vue québécois, cette analyse suscitera sans doute des réactions divergentes. On peut penser que les souverainistes se réjouiront non seulement de la sérénité avec laquelle la souveraineté du Québec est envisagée, mais aussi du constat qui se dégage concernant l'état anémique des relations entre le Québec et le reste du Canada, qui tendent à conforter leur option. Il est peu probable que les fédéralistes partagent cet enthousiasme pour les raisons inverses.

Il faut dire cependant que cet aspect du projet n'est sans doute pas la motivation première des participants. Dans tous les cas, il tient la part congrue de l'analyse et, parfois, il laisse place aux pires idées reçues. À ce titre, la décision de Frank Davey de définir le Québec, «...culturally, linguistically and territorially, in that order, as the francophone culture of roughly the southern half of Quebec province» (p. 119) est exemplaire. C'est d'abord le 
désir de faire le point sur la situation du reste du Canada qui donne à l'ouvrage sa véritable valeur.

Programme d'études sur le Québec Université McGill

SARAH FORTIN 